\title{
BENT SOLENOIDS FOR SPECTROMETERS AND EMITTANCE EXCHANGE SECTIONS *
}

\section{J. Norem ${ }^{\dagger}$, Argonne National Laboratory, Argonne IL, 60439 USA}

\section{Abstract}

Bent solenoids can be used to transport of low energy beams as they provide both confinment and dispersion of particle orbits. Solenoids are being considered both as emittance exchange sections and spectrometers in the muon cooling system as part of the study of the muon collider. We present the results of a study of bent solenoids which considers the design of coupling sections between bent solenoids to straight solenoids, drift compensation fields, aberrations, and factors relating to the construction, such as field ripple, stored energy, coil forces and field errors.

\section{INTRODUCTION}

While the optics of beams in straight solenoids is well understood in accelerator physics[1], and the behavior of charged particles in toroidal geometries is also well understood in plasma physics[2], the optics of beams which traverse both bent and straight sections have not had significant applications in either field, are not simple or symmetric, and are not particularly well understood[3]. Beams in bent solenoids are subject to drifts perpendicular to thebend plane. The basic geometry is shown in Fig. 1 .

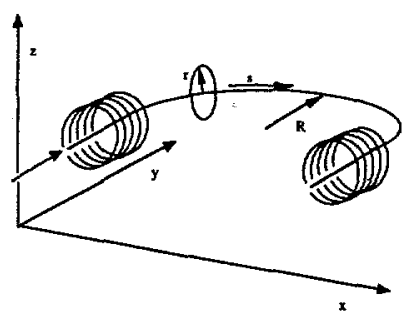

Figure 1, A bend without coupling sections.

In a straight solenoid the magnetic field is homogeneous, while in a bend the field is distributed according to $1 / R$, this means that there is a transition region where the field is difficult to parametrize and should be calculated numerically see Figure 2.

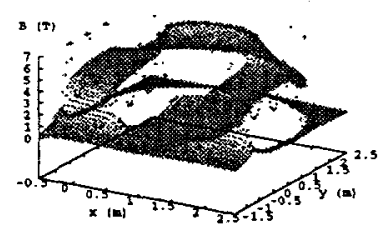

Figure 2, The field in a bent solenoid
As a result this complexity there are a large number of variables associated with the design of a solenoidal bend. In addition to the nominal field on axis, bend radius, coil radius and bend length, there is the choice of field to compensate perpendicular drifts, the bending algorithm used to couple the bend to the straight section, and the algorithm used to compare the field in the bent and straight sections.

\section{BEND BASICS}

A particle passing through the magnet system described in Figures 1 and 2 will be mismatched to the bend and execute an orbit like that shown in figure 3 , where the centrifugal momentum drives the particle to larger radii, produces an upward drift and excites Larmor oscillations at the end of the bend.

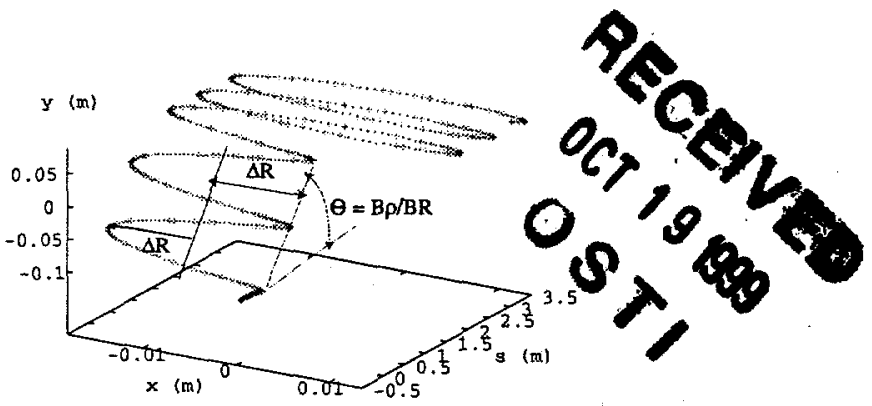

Figure 3, particle orbits in bent solenoids, where $x$ and $y$ are perpendicular to the particle motion.

The vertical drift is described by the relations

$$
\begin{aligned}
& \mathbf{v}_{c}=\frac{m_{0} v_{\|}^{2}}{q B^{2}} \frac{\mathbf{R} \times \mathbf{B}}{R^{2}} \\
& \mathbf{v}_{\nabla B}= \pm \frac{1}{2} v_{\perp} r_{L} \frac{\mathbf{B} \times \nabla B}{B^{2}} \\
& \mathbf{v}_{\text {drift }}=\mathbf{v}_{c}+\mathbf{v}_{\nabla B}=\frac{m_{0}}{q} \frac{\mathbf{R} \times \mathbf{B}}{B^{2} R^{2}}\left(v_{\|}^{2}+0.5 v_{\perp}^{2}\right) \\
& \theta_{\text {drift }}=B \rho / B_{\|} R-B_{\perp} / B_{\|} \\
& \Delta R=(B \rho)_{d r i f t} / B=(B \rho)^{2} / B^{2} R
\end{aligned}
$$

where Larmor length is $L=2 \pi B \rho / B$, and $B \rho$ is the magnetic rigidity.

\subsection{Compensating Fields}

The vertical drift can be eliminated with a compensating field $B_{\perp}$ which provides a negative slope of the magnetic

\footnotetext{
' norem@hep.anl.gov

To be presented at the 1999 Particle Accelerator Conference (PAC'99), New York, New York, March 29 to April 02, 1999.
} 


\section{DISCLAIMER}

This report was prepared as an account of work sponsored by an agency of the United States Government. Neither the United States Government nor any agency thereof, nor any of their employees, make any warranty, express or implied, or assumes any legal liability or responsibility for the accuracy, completeness, or usefulness of any information, apparatus, product, or process disclosed, or represents that its use would not infringe privately owned rights. Reference herein to any specific commercial product, process, or service by trade name, trademark, manufacturer, or otherwise does not necessarily constitute or imply its endorsement, recommendation, or favoring by the United States Government or any agency thereof. The views and opinions of authors expressed herein do not necessarily state or reflect those of the United States Government or any agency thereof. 


\section{DISCLAIMER}

Portions of this document may be illegible in electronic image products. Images are produced from the best available original document. 
field lines so that $\theta_{d r i f}=B \rho / B_{\| 1} R-B_{\perp} / B_{\|}=0$. Thus a single momentum can be confined to a plane, while other momenta will be subject to the same momentum dependence, giving a dispersion

$$
\dot{D}=\theta_{\text {drift }} s=\int B \rho / B_{\|} R d s \sim \Theta B \rho / B .
$$

The vertical field can be applied either with an external coil set or by tipping the coils. An advantage of tipping the coils is that the vertical field component can be tailored to the local bend radius. The coils would be tipped as shown in figure 4

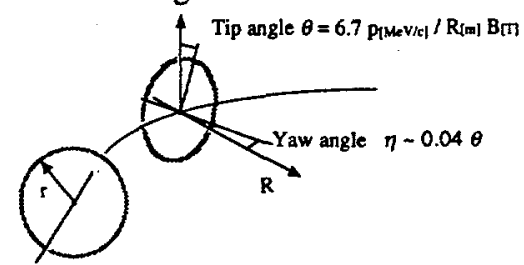

Figure 4, Tipping and turning coils.

\section{COUPLING SECTIONS}

It is possible to minimize the mismatch where straight meets bend by using a veriety of coupling sections. The mismatch can be caused by two effects: 1) the orbit cannot adjust smoothly to the position of the equilibrium orbit as it goes through the bend, and, 2) the magnetic field lines are displaced from what would be the particle orbit by $1 / R$ (or other) effects. Both of these effects can minimized using a good coil design.

\subsection{Adiabatic Bends}

If the mismatch that causes the oscillations in Figure 3 is spread out over many Larmor lengths and phases, the transverse oscillations will be cancelled, unfortunately at the cost of a long solenoid.

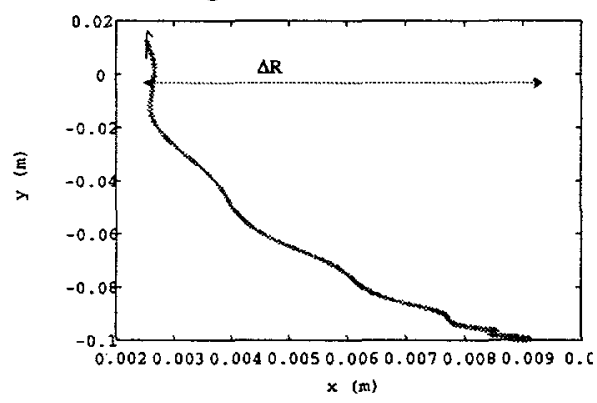

Figure 5, A two dimensional projection of the motion of a particle in an adiabatic coupler.

\section{$2.2 L=n \lambda_{\mathrm{L}}$}

If the overall bend length is adjusted to be equal to an even number of Larmor oscillations, the particle can exit from the bend at the point of the cusp (see figure 3 ) where it has no momentum perpendicular to the direction of motion. This method had two problems: 1) the length of the magnet is fixed and, 2) effects due to coil size can complicate the effective length of the bend section. Nevertheless, for small dispersion systems this solution can be very useful.

\section{$2.3 L=\lambda_{\mathrm{L}} / 2$ Coupler}

While the mismatch produced at discontinuities is generally a problem, it is possible to use the mismatch constructively to create a half Larmor oscillation of the correct size and length to couple into a bend of arbitrary radius. This can be done by designing a coupling section with half the bend radius of the primary bend and a length equal to $L=\lambda_{L} / 2$. The coupling section then brings the particle to what would be its equilibrium offset $\Delta R$ in the bend without residual perpendicular momentum. The use of this technique is complicated by coil radius effects which can complicate evaluating the section length, causing mismatch at the ends of the sections.

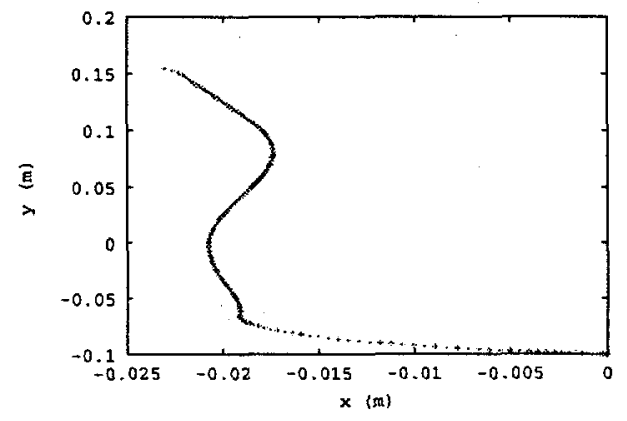

Figure 6. A particle orbit in a $L=\lambda_{\mathrm{L}} / 2$ coupler, showing mismatch at the end of the section.

\subsection{Short Smooth Couplers}

An extension of the $L=\lambda_{L} / 2$ coupler is to use a smoothed curve which has the advantages that discontinuities in both $\mathrm{d} \Theta / \mathrm{d} s$ and its derivatives are avoided. The expression used by Fernow and Palmer[4],

$$
\frac{d \Theta}{d s}=0.5\left\{\cot \left(\frac{s-s_{1}}{\sigma}\right)-\cot \left(\frac{s-s_{2}}{\sigma}\right)\right\},
$$

where the length over which the transition is made $\sigma=$ $\lambda_{1} / 2$ seems to be optimum, consistent with the arguments presented in Sections 3.1 and 3.3, see Figure 7.

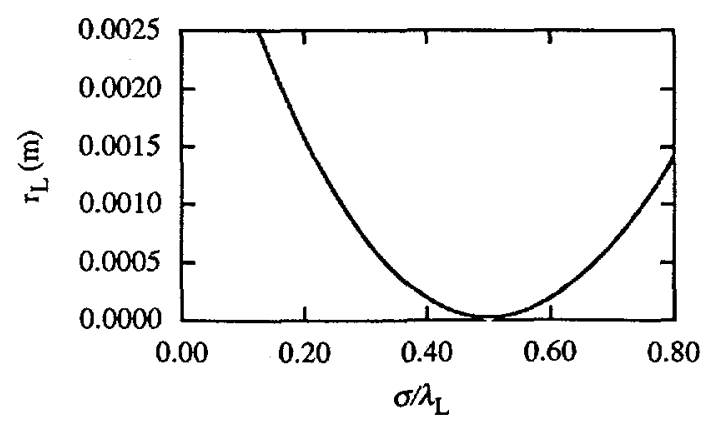

Figure 7, Mismatch produced as a function of short, smooth coupler length. 


\section{ABERRATIONS}

$\mathrm{Tn}$ addition to the time slewing due to the longer path traveled by particles on the outside of a bend, there are a number of aberrations which complicate the optics.

\section{$4.1 x^{\prime} y^{\prime}$ Dependence}

Particles with momentum perpendicular to the beam will drift vertically due to grad $B$ drift. This can be eliminated with equal numbers of bends of opposite direction, but in most cases with $x^{\prime}<0.3$ the drift is small, comparable to a $\delta p / p \sim 2 \%$. This is shown in Figure $\mathrm{X}$

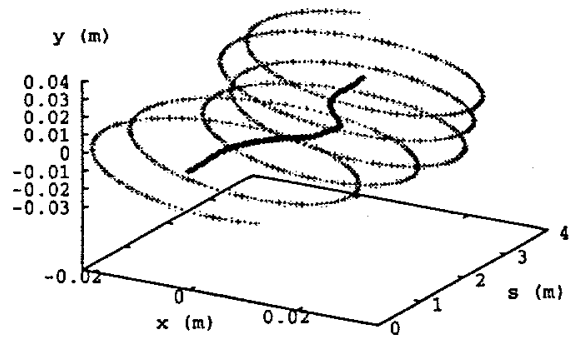

Figure X, Grad B drift for orbits with $\mathrm{p}_{\perp} \neq 0$.

\subsection{Shears}

Even if all translation of the central orbit is canceled using a good coil design the orbits off center are subject to a shear. This shear is a result of mismatches between the ideal compensation field and that provided by physical coils. The effects of this sheer are shown if Fig Y, and the shear can be eliminated by equal right and left bend angles.

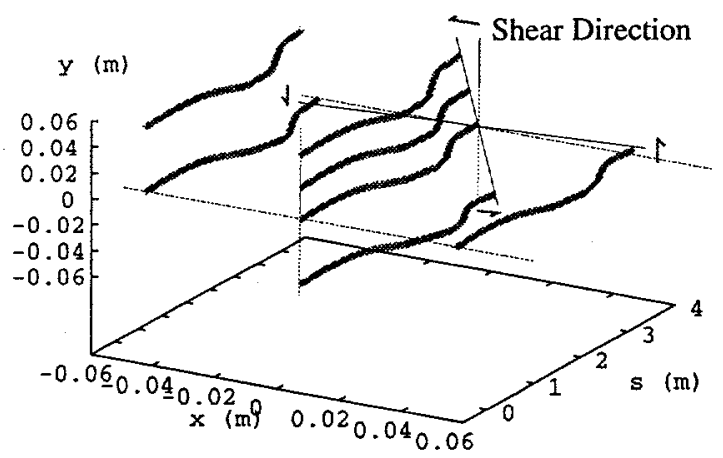

Figure $Y$, Shears in large beams over a bend.

The sum of horizontal and vertical components of the shear should be constant because the perpendicular field components that cause it are determined by Amperes. law, integrating around the path shown at right. The relative

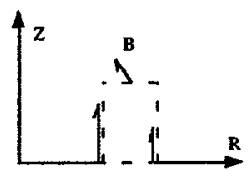
amplitude of horizontal and vertical shear can be altered by altering the geometry of the coils causing the compensating field.

\subsection{Coil Geometry Effects}

The coil geometry interacts strongly with the optics, as shown in figure $Z$ below, which looks at an orbit for two differrent coil radii.

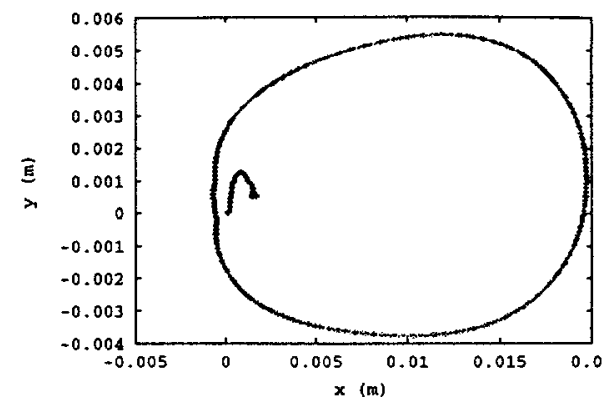

Figure Z, Orbits for coil radii $r=0.3$ and 0.03 .

Reference GPT

\section{CONCLUSIONS}

\section{REFERENCES}

The submitted manuscript has been created by the University of Chicago as Operator of Argonne National Laboratory ("Argonne") under Contract No. W-31-109-ENG-38 with the U.S. Department of Energy. The U.S. Government retains for itself, and others acting on its behalf, a paid-up, nonexclusive, irrevocable worldwide license in said article to reproduce, prepare derivative works, distribute copies to the public, and perform publicly and display publicly, by or on behalf of the Government. 